See discussions, stats, and author profiles for this publication at: https://www.researchgate.net/publication/3957619

\title{
A general approach to nonrigid registration: decoupled optimization
}

Conference Paper · February 2001

DOI: 10.1109/IEMBS.2001.1017266 · Source: IEEE Xplore

CITATIONS

4

5 authors, including:

Eduardo Suarez-Santana

Instituto Tecnológico de Canarias

19 PUBLICATIONS 271 CITATIONS

SEE PROFILE

Carlos Alberola-López

Universidad de Valladolid

240 PUBLICATIONS 2,843 CITATIONS

SEE PROFILE
READS

18

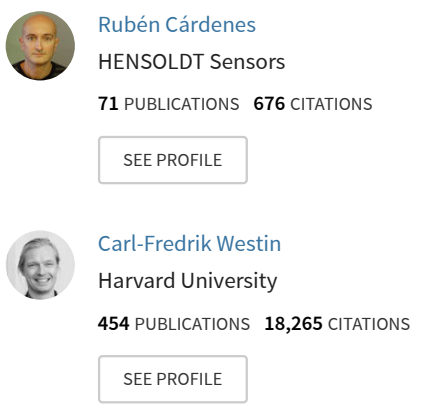

Some of the authors of this publication are also working on these related projects:

Sparse deconvolution of higher order tensor for fiber orientation distribution estimation View project

Free Water Imaging View project 


\title{
A GENERAL APPROACH TO NONRIGID REGISTRATION: DECOUPLED OPTIMIZATION
}

\author{
Eduardo Suárez ${ }^{1}$, Rubén Cárdenes ${ }^{1}$, Carlos Alberola ${ }^{2}$, C.-F. Westin ${ }^{3}$, Juan Ruiz-Alzola ${ }^{1}$ \\ ${ }^{1}$ Dep. Señales y Comunicaciones. Universidad de Las Palmas de Gran Canaria, SPAIN \\ ${ }^{2}$ Dep. Teoría de la Señal,Universidad de Valladolid, Valladolid, SPAIN \\ ${ }^{3}$ Harvard Medical School and Brigham \& Women's Hospital, Dept. Radiology, Boston, USA
}

\begin{abstract}
To a great extent, the success of advanced image-guided medical procedures hinges on non-rigid volume registration. For example, nonrigid registration must be applied in interventional approaches where intraoperative information is used to update high-quality preoperative data; in follow-up studies in order to assess time-evolution of development; aging, pathology or treatment; and in many other applications including intersubject variability and population-based atlas construction.

In this paper we examine several computational schemes that warps one volumetric dataset onto another. We also explore the inevitable trade-off between the computational load and the incorporation of sophisticated similarity measures necessary for multimodal volumes. Estimated deformation fields are based on the variational formulation of Partial Derivative Equations (PDEs), which includes a similarity and a regularization term. We compare numerical solutions to this problem using the Euler-Lagrange equations (EL), the Finite Elements discretization (FE), and Decoupled Optimization over the possible deformations (DO).
\end{abstract}

Keywords-Registration, Correlation Coefficient, Finite Elements Method, Euler-Lagrange, Template Matching, Optimization

\section{INTRODUCTION}

Advanced computer-assisted interventions require 3D warping methods to perform non-rigid registrations among different volume datasets, an essential feature of interventional procedures [1] where preoperative data and models must be updated using intraoperative imaging to successfully manage natural and induced alterations of anatomy. In cases such as these, time consuming image acquisition and processing is curtailed by operating room procedures, and this obviously limits the guidance that intraoperative images can provide. In an effort to overcame this obstacle, we suggest warping high quality preoperative data and models into the coordinate system of intraoperative real anatomy (scanned by an interventional imaging device), which, in turn, is used to guide the surgical procedure. The warp is obtained by estimating a deformation field and then non-rigidly adapting the proeoperative data to the interoperative data by non-rigidly registering the preoperative and intraoperative datasets. Additional applications include follow-up studies of the same individual by non-rigid registration of image data (e.g., developmental [2] and aging effects [3] studies, degenerative pathology [4] and treatment [5] evolution). Nonrigid registration is also crucial to intrasubject comparison (i.e., in the construction of population-based atlas [6] where datasets from different individuals must be related to a canonical frame). Further, non-rigid registration must incorporate a priory anatomic knowledge into automatic segmentation algorithms, such as in the framework of template-driven segmentation proposed by Warfield et al. [7].

Several approaches have been proposer for volume data registration that rely on a multi-resolution decomposition of the volumes. With regard to non-rigid registration algorithms, the formulas must recognize potential matches based on the similarity of the neighborhoods to corresponding points in both datasets. From a mathematical standpoint, these algorithms are typically encoded in such a way that similarity functions between neighborhoods are defined. In general, many combinations are possible for acceptable levels of the similarity function , although the inverse problem of estimating the deformation field between both datasets is ill-conceive, a typical problem in regularization theory as defined. For example, to constrain the number of so- lutions into a single one solution.

An optimal way of including both the similarity and the regularization conditions in an operating equation is to use models based on variational formulations and on PDEs. For example, the well-known sum of squared differences similarity function is used in [8] with a viscous fluid constraint that does not need any small deformation assumption, in [9] with an elasticity constraint, solving the PDE with the FE method. Dengler and Schmidt [10] proposed an optical flow method with an elasticity constraint, solved with a multiresolution pyramid with FEM discretization (utilizing the sign of Laplacian pyramid as the matching features). Another useful similarity measure is the correlation index, which considers neighborhoods to be similar if their intensities are related by an affine function. This measure was applied in [11] with an elasticity constraint as regularization; solving the PDE with the Jacobi method; unlikewise in [12] but solving the PDE iteratively with FE.

Though there has been significant efforts to solve the nonrigid registration problem, there has been relatively little research into more complex similarity functions, such as the mutual information broadly used for rigid registration [13], which are essential for multimodal registration. However, arbitrary similarity functions (see [14] for examples) are difficult to be incorporated into the computational algorithms due to their lack of mathematical tractability. This deficiency, fortunately, can be compensated for by directly computing the similarity (as opposed to applying a an analytical derivation of the similarity function). In this paper, we will frame the variational problem as one of "equivalent optimization", and solve it by determining the set of voxel displacements. We will the compare these results with those obtained using the Euler Lagrange equations and Finite Elements discretizations.

\section{VARIATIONAL FORMULATION}

A common approach to nonrigid registration lies in establishing the displacement field $\vec{d}(\vec{r})$ which minimizes an energy functional with a similarity and a regularization term relating to reference and current volumes; that is,

$$
\mathcal{J}(\vec{u}(\vec{r}))=\int_{\mathbf{D}}[\mathcal{S}(\vec{r}, \vec{u}(\vec{r}))+\mathcal{R}(\vec{r}, \vec{u}(\vec{r}))] d \vec{r}
$$

where $\mathcal{S}$ is the similarity measure and $\mathcal{R}$ is the regularization term. $\mathcal{R}$ is usually a smoothness constraint.

In order to make comparisons, we have chosen the correlation coefficient as the similarity function and the sum of squared partial derivatives of the displacement as the regularization constraint. Hence, the correlation coefficient between two random variables $x$ and $y$ is defined as:

$$
C C(x, y)^{2}=\left[\frac{\sigma_{x y}}{\sigma_{x} \sigma_{y}}\right]^{2}
$$

Where $\sigma_{x y}$, is the cross covariance and $\sigma_{x}, \sigma_{y}$ are the standard deviations of $x$ and $y$ respectively. Inherit in the non-rigid registration problem, are the intensities of two tentatively corresponding voxels $x$ and $y$. The correlation coefficient is estimated from two windows centered on those voxels. However, 


\section{Report Documentation Page}

\begin{tabular}{|l|c|c|}
\hline $\begin{array}{l}\text { Report Date } \\
\text { 25OCT2001 }\end{array}$ & $\begin{array}{c}\text { Report Type } \\
\text { N/A }\end{array}$ & Dates Covered (from... to) \\
\hline
\end{tabular}

\section{Title and Subtitle}

A General Approach to Nonrigid Registration: Decoupled Optimization

Author(s)

Performing Organization Name(s) and Address(es)

Dep. Senales y Communicaciones Universidad de Las Palma de Gran Canaria, Spain

Sponsoring/Monitoring Agency Name(s) and Address(es)

US Army Research, Development \& Standardization Group

(UK) PSC 802 Box 15 FPO AE 09499-1500
Contract Number

Grant Number

Program Element Number

Project Number

Task Number

Work Unit Number

Performing Organization Report Number

Sponsor/Monitor's Acronym(s)

Sponsor/Monitor's Report Number(s)

\section{Distribution/Availability Statement}

Approved for public release, distribution unlimited

\section{Supplementary Notes}

Papers from the 23rd Annual International Conference of the IEEE Engineering in Medicine and Biology Society, October 25-28, 2001, held in Istanbul, Turkey. See also ADM001351 for entire conference on cd-rom., The original document contains color images.

\section{Abstract \\ Subject Terms \\ Report Classification \\ unclassified}

Classification of Abstract

unclassified

Number of Pages

4
Classification of this page

unclassified

\section{Limitation of Abstract}

UU 
window size must be traded off in order to be local and to harvest a sufficient number of samples for a meaningful estimation. The correlation coefficient facilitates similarity among voxels if the intensities inside are related through an affine function.

For the regularization term, we use

$$
R=|\nabla \otimes \vec{u}(\vec{r})|_{\text {fro }}^{2} 1
$$

where $\vec{u}$ is the deformation field, and which is extensively used in optical flow applications [15] and essentially imposes a smoothness constraint on the deformation field.

\section{DECOUPLED OPTIMIZATION}

From a numerical point of view, the minimization of the functional (1), i.e., the estimation of the displacement field, can be viewed as an optimization problem, since the displacement vectors for every voxel are the parameters that must be optimized. Considered in this way, the functional becomes a function or cost of the set of displacements; hence, the minimization of the functional is equivalent to the minimization of a multi-variate cost. This relationship, in turn, allows us to conduct a numerical search for the minimum, thus avoiding the mathematical intractability of complicated functionals. Nevertheless, optimization is a daunting task that requires a clever approach. Notice the number of unknown parameters is three times (in 3D) the number of voxels and the highly nonlinear nature of the functionals with nontrivial similarity or regularization terms.

In this paper, we propose here a numerical method, general enough to deal with any similarity function or regularization term based on the minimization of (1) considered as a cost. In order to avoid getting stuck embedded in local minima, and to accelerate execution time, we have developed a feasible, initial solution to the optimization scheme. To this extent, we first compute a displacement field by local template matching on high structure points and subsequent interpolation, embbeding the approach into a gaussian pyramid. Our implementation relies on part of our previous work, where the structure detection is based on the eigenanalysis of the correlation matrices of the gradient of the datasets at every point and we use a Kriging [16] estimator to make the interpolation [17], [18]. It is interesting to note that this approach is able to deal with general tensor data, such as diffusion tensor MRI and hence it allows the generalization of the method proposed here to tensor data. Nevertheless, for the purposes of this paper, any template matching method which is able to provide an initial solution can be used.

The number of dimensions of a 3-d displacement field search space is three times the number of voxels. In order to save computational cost, we decouple the problem searching for local minima at each voxel. The optimization algorithm used is based on a Quasi-Newton technique called BFGS [19] . Separate minimization of each voxel needs knowledgment of neighbor voxels, so minimization of every voxel doesn't provide us the nearest local minimum in the global search space. To avoid this, several iterations to the whole dataset are carried out until minimization reaches a consistent solution. To avoid false optimizations that the regularization term cannot deal, a median filter on each deformation field component is carried out as the initial field for the next iteration.

\section{Two Alternative Methods}

In what follows we compare the proposed method with the Euler-Lagrange approach and with a Finite Element discretization. A brief introduction to these two methods is given below. Both methods are implemented using a gaussian pyramid.

\footnotetext{
${ }^{1}$ Frobenuis norm
}

\section{A. Euler-Lagrange}

The Euler-Lagrange Method consists of applying variational calculus to equation (1), and obtaining a list of differential equations which can be solved iteratively. This procedure is a difficult task for non-trivial similarity measures and regularization terms. In the case we have described, the similarity measure and the regularization term have been approximated to first and second order of $\vec{u}$.

Using Euler-Lagrange equations for the energy functional (1), we reach this linear system of equations, defined as

$$
\begin{array}{r}
a_{x}+2\left(A_{11} u^{n+1}+A_{12} v^{n+1}+A_{13} w^{n+1}\right)=2 \alpha^{2}\left(\bar{u}^{n}-u^{n+1}\right) \\
a_{y}+2\left(A_{21} u^{n+1}+A_{22} v^{n+1}+A_{23} w^{n+1}\right)=2 \alpha^{2}\left(\bar{v}^{n}-v^{n+1}\right) \\
a_{z}+2\left(A_{31} u^{n+1}+A_{32} v^{n+1}+A_{33} w^{n+1}\right)=2 \alpha^{2}\left(\bar{w}^{n}-w^{n+1}\right)
\end{array}
$$

where $a_{i}$ and $A_{i j}$ are the first and second order coefficients of the similarity measure respectively. In matrix form this can be depicted as

$$
\vec{a}+2 \mathcal{A} \vec{u}^{n+1}=2 \alpha^{2}\left(\vec{u}^{n}-\vec{u}^{n+1}\right)
$$

This equation can be solved by the iterative Gauss-Seidel Method in which the updated components of the deformation field $\left(u^{n+1}, v^{n+1}, w^{n+1}\right)$ are computed from $\left(u^{n}, v^{n}, w^{n}\right)$ in every step using

$$
\vec{u}^{n+1}=\left(\mathcal{A}-\mathcal{I} \alpha^{2}\right)^{-1}\left(\vec{u}^{n}-\frac{1}{2} \vec{a}\right)
$$

\section{B. Finite Elements}

The Finite Elements method [20] relies on interpolating the solution using as nodal points the vertices of a discretization of the domain, by means of a linear formulation where the values at the nodal points are the unknowns.

In the $3 \mathrm{D}$ case, we have selected a tetrahedral mesh; and in the $2 \mathrm{D}$ case, we have chosen a triangular mesh. The refinement of the mesh is chosen as a function of the level of detail we have assumed. In a multi-resolution scheme, we generate a mesh with very few triangles at the top level of the resolution pyramid and increase the number of triangles as we move to higher resolutions.

In order to accelerate the mesh generation, we will assign bigger elements to less detailed zones and smaller ones to high information regions of the dataset.

The algorithm has been implemented using first and second order approximations of the correlation coefficient. Since the results derived from first and second order approximations are not significantly different, we will only present results from first order approximations.

\section{Results}

In this section we present the results for the registration of several medical datasets. To compare the performance, we have used the same similarity measure and the same regularization term.

Figure 1a shows a small region of an axial proton density (PD) MR image and fig. 1b shows the same region in a T2 weighted image, though slightly deformed by a synthetic field. The synthetic field has been generated by smoothing a random initial vector field. Figure 2 a shows a detail of the synthetic deformation field. Figure $2 \mathrm{~b}$ shows the deformation field registered by using the Euler-Lagrange method, with $\alpha=0.8$, window size $w=5$, using 20 iterations and initialized with the zero solution. Figure $2 \mathrm{c}$ shows the deformation field registered by using the finite elements method, with $\alpha=0.8$, window size $w=5$, and a triangular mesh of 312 triangles and 177 nodes. Finally, fig. 2d 
shows the deformation field registered by using decoupled optimization, where the initial solution has been obtained using template matching with a search window of size $w_{\text {search }}=5$ and a similarity window of size $w_{\text {similarity }}=3$. The local optimization has been constrained to an amplitude of five pixels, $\alpha=0.8$, and using 7 iterations to the whole dataset.

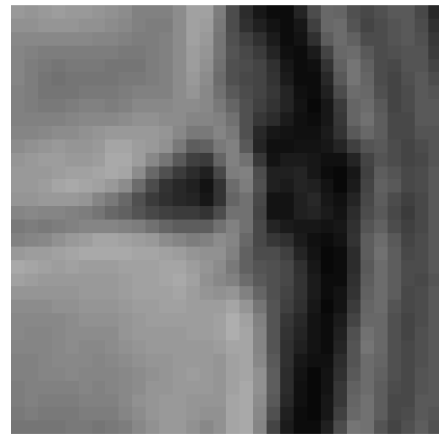

a)

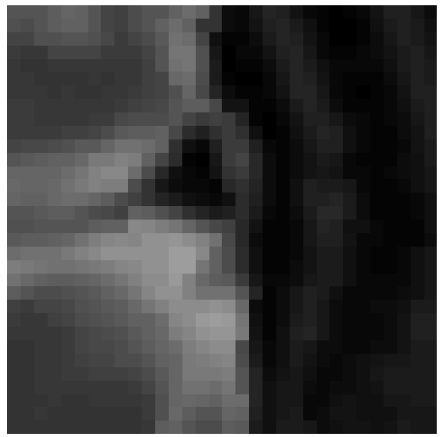

b)
Fig. 1. MRI images PD (a) and T2 (b)

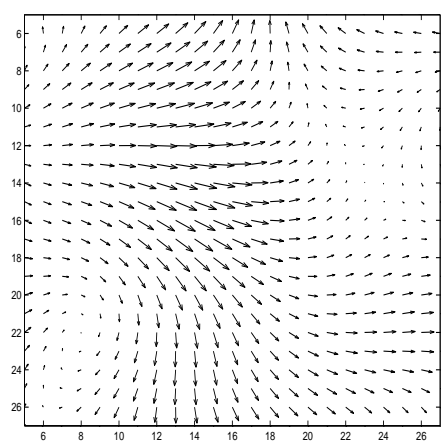

a)

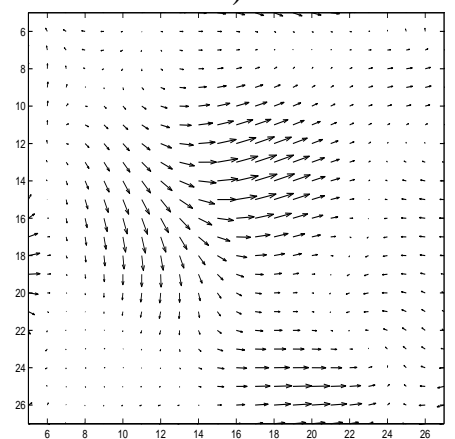

c)

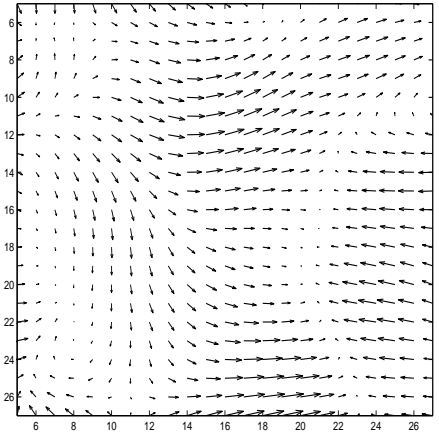

b)

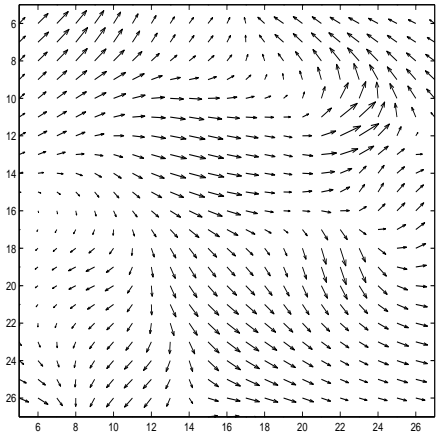

d)
Fig. 2. Synthetic deformation field (a) and deformation fields obtained with Finite Elements (b), Euler-Lagrange (c) and optimization(d)

Figure 3a shows an overlay of T1w and T2w MR images corresponding to two different patients and the surface models of their ventricles. Figure $3 \mathrm{~b}$ shows the estimated deformation field using the decoupled optimization approach overlaid onto the models. The yellow ventricle is considered to be the reference, and the green ventricle is considered to be the current.

In order to compare the results obtained by the three methods, fig. 4 shows a detail of one coronal section of the reference T1 image and the vector fields on that section will be projected onto it. Figure $4 b-d$ shows the results obtained for the three methods, EL (a), FE (b) and DO (c), with similar parameters to those used in the previous experiment.
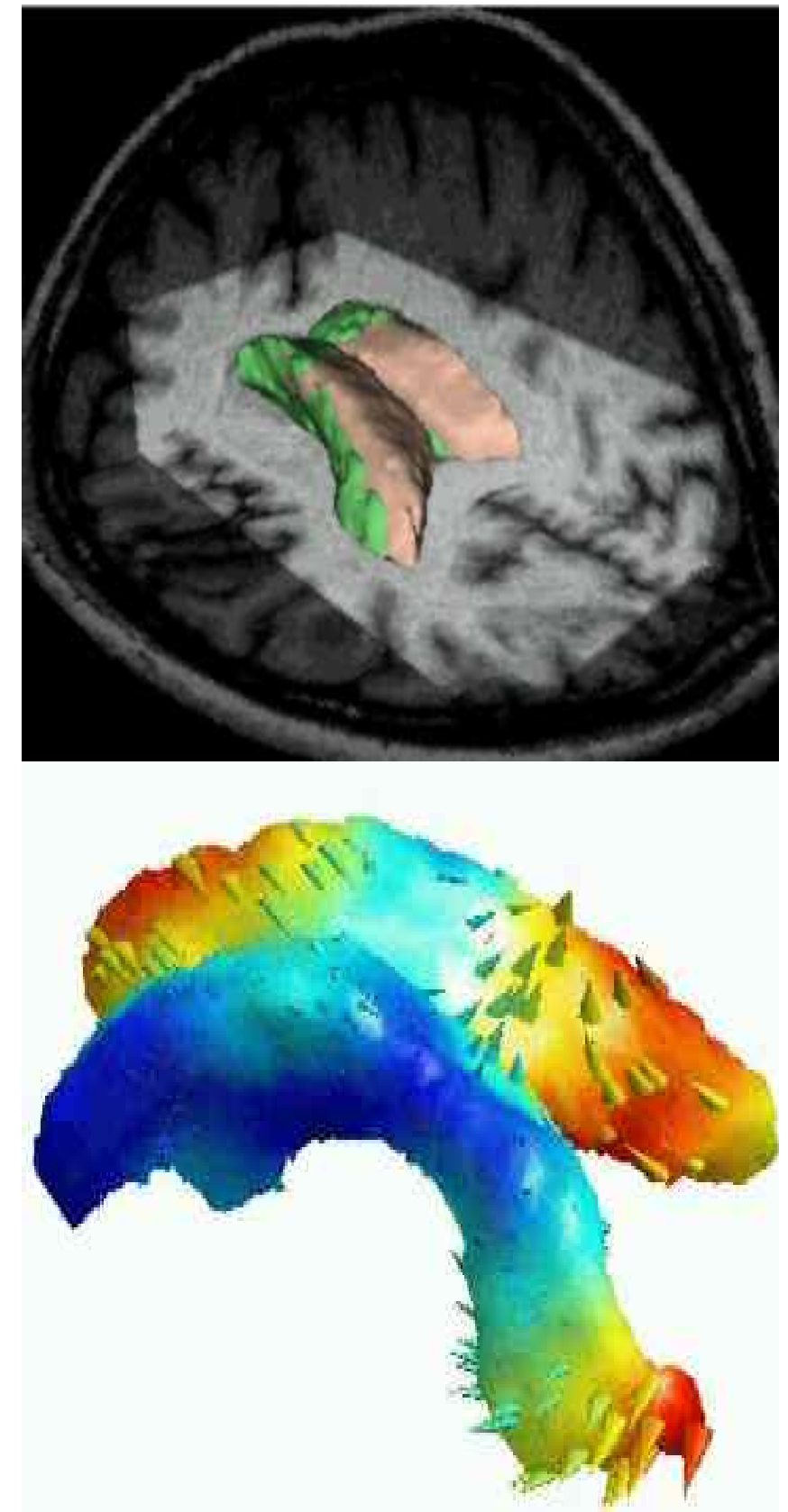

Fig. 3. Above: ventricle models generated with the MRI T1 (green) and with the MRI T2 (orange) from two different patients and original images. Below: Three-dimensional deformation field overlaid on a ventricle model. Hot colors means large deformations

As a measure of quality, the absolute correlation coefficient has been computed in different cases, which summarizes table 1. Results for a synthetic image not shown are also displayed.

\section{CONCLUSIONS AND FURTHER RESEARCH}

In this paper we have introduced a novel approach to general nonrigid registration problems based on Decoupled Optimization (DO) solution to a variational formulation and it has been compared with two other solutions to the same variational problem: Euler-Lagrange (EL) and Finite Elements (FE). Although the approaches are quite different in nature, they all give similar and meaningful results when used with Correlation Coefficient as the similarity measure and eq. (3) as the regularization term. The main differences between these methods are related to 


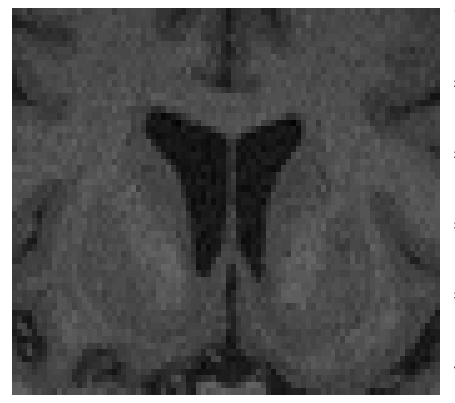

a)

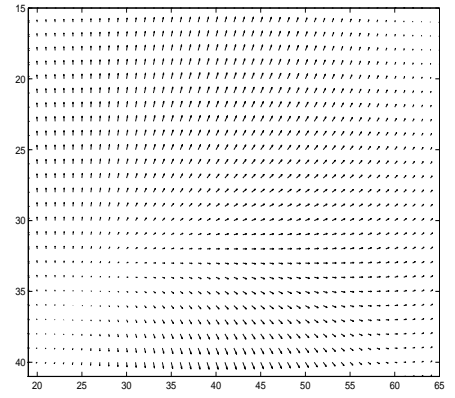

c)

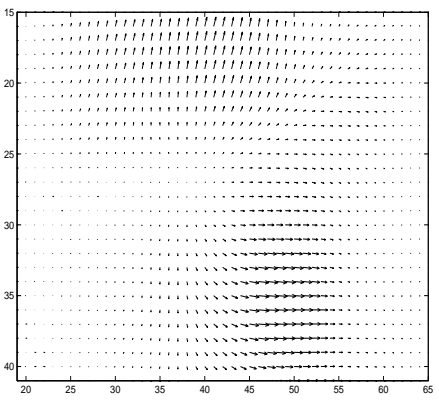

b)

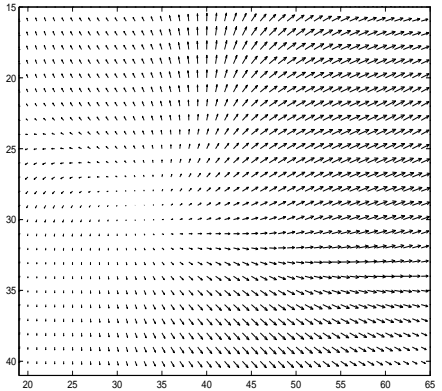

d)
Fig. 4. T1 MRI coronal section (a) and deformation fields obtained with EulerLagrange (b), Finite Elements (c) and direct optimization (d)

TABLE I

ABsolute CorRelation COEFFicients

\begin{tabular}{||c||c|c|c||}
\hline Registration & Synthetic & T2-PD & T1-T2 \\
\hline Not deformed & 1 & 0.76 & 0.73 \\
EL & 0.84 & 0.71 & 0.69 \\
FE & 0.80 & 0.70 & 0.65 \\
DO & 0.85 & 0.73 & 0.70 \\
\hline
\end{tabular}

computational cost, and possibility of incorporating other similarity measures or regularization terms. The computational cost of Euler-Lagrange is smaller than Finite Elements and much smaller than Optimization. Nevertheless, the DO method allows an easy incorporation of any similarity measure, such as mutual information based ones, or regularization term. Moreover, its extension to deal with multivalued data is also possible, as it uses algorithms developed to deal with tensor data in order to get good initial deformation fields and the optimization solution is powerful enough to incorporate the tensor reorientations. Nevertheless, this is still something we are investigating and that will be reported in a forthcoming paper.

Other research efforts in this area are focused on comparing with other optimization algorithms, such as for example stochastic, more efficient in terms of computational load and with the ability of jumping out local optima, and in high performance implementations using clusters of low cost personal computers (beowulfs). Our motivation is to obtain a robust and efficient computational tool which can be easily reconfigured to deal with different registration problems in a clinical environment.

\section{REFERENCES}

[1] F. Jolesz, A. Nabavi, and R. Kikinis, "Integration of interventional mri with computer assisted surgery," 2001.

[2] P. Huppi et al., "Quantitative magnetic resonance imaging of premature and mature newborns," 1998.

[3] C. Guttman et al., "White matter changes with normal aging," 1998.
[4] Kikinis. R. et al., "Quantitative follow-up of patients with multiple sclerosis using mri: Technical aspects," 1999.

[5] C. Guttman et al., "Computerized image processing for quantitative follow-up of patients," 1999.

[6] J.C. Mazziotta et al., "A probabilistic atlas of the human brain: Theory and rationale for its development," 1995.

[7] Arthur W. Toga, Brain Warping, chapter 4, pp. 67-84, Academic Press, 1999.

[8] G.E. Christensen, Deformable Shape Models For Anatomy, Ph.D. thesis, Washington University, 1994.

[9] M. Ferrant, S.K. Warfield, C.R. Guttman, R.V. Mulkern, F. Jolesz, and R. Kikinis, "3d image matching using a finite element based elastic deformation model," in Medical Image Computing and Computer-Assisted Intervention, 1999.

[10] J. Dengler and M. Schmidt, "The dynamic pyramid a model for motion analysis with controlled continuity," 1988.

[11] R. Bajcsy and I. Kovacic, "Multiresolution elastic matching,".

[12] J. Gee, C. Barillot, L.L. Briquer, D. Haynor, and R. Bajcsy, "Matching structural images of the human brain using statistical and geometrical image features," in SPIE. Visualization in Biomedical Computing, 1994, vol. 2359, pp. 191-204.

[13] W. Wells et al., "Multimodal volume registration by maximization of mutual information," 1996.

[14] A. Roche et al., "Towards a better comprehension of similarity measures used in medical image registration," in Medical Image Computing and Computer-Assisted Intervention, 1999 , pp. 555-566.

[15] B.K.P. Horn and B.G. Schunk, "Determining optical flow," 1986.

[16] J. Ruiz-Alzola, R. Kikinis, and Westin C.-F., "Detection of point landmarks in multidimensional tensor data," .

[17] J. Ruiz-Alzola, C.F. Westin, Warfield S.K., A. Nabavi, and R. Kikinis, "Nonrigid registration of 3d scalar, vector and tensor medical data," in Third International Conference On Medical Robotics, Imaging and Computer Assisted Surgery, 2000, pp. 541-550.

[18] J. Ruiz-Alzola et al., "Nonrigid registration of $3 \mathrm{~d}$ tensor medical data," .

[19] J.E. Dennis and R.B. Shnabel, Numerical Methods for Unconstrained Optimization and Nonlinear Equations, Prentice Hall, 1983.

[20] O.C. Zienkewickz and R.L. Taylor, The Finite Element Method, McGraw Hill Book Co., 1987. 